\title{
Endoscopic closure of gastric perforation from eroded Jackson- Pratt drain using over-the-scope clips
}

\section{Sofia Nigar, Emmanuel Ofori, Daryl Ramai, Mahesh Krishnaiah}

The Brooklyn Hospital Center, Academic Affiliate of The Icahn School of Medicine at Mount Sinai, Clinical Affiliate of The Mount Sinai Hospital, Brooklyn, NY, USA

A 55-year-old woman with a past medical history of hypertension, diabetes mellitus, hyperlipidemia, and atrial fibrillation (on rivaroxaban) presented with a abdominal pain. Her past surgical history was significant for prior gastric bypass. Computed tomography scan showed a ventral hernia with several non-inflamed small bowel loops, pneumoperitoneum, and changes at the gastric bypass site including edematous and fluid-filled stomach remnant. An explorative laparotomy was performed and revealed an incarcerated omental incisional hernia. Gastrojejunostomy resection was performed, with the creation of a new gastrojejunostomy and enterotomy of the alimentary limb. A Stamm gastrostomy was performed in the remnant stomach. Postoperatively, the patient complained of abdominal pain, and an upper endoscopy was performed, which showed that the Jackson-Pratt (JP) drain had perforated into the stomach lumen through the staple line (Fig. 1A). Under endoscopic guidance, the JP drain was withdrawn until it was no longer visible in the stomach lumen and was then secured to the skin (Fig. 1B). The perforation was repaired using over-the-scope clips (OTSCs) (Fig. 2A, B). After 14 days, repeat esophagogastroduodenoscopy showed the OTSCs approximating the mucosa, and a swallow study showed no leakage.

Department of Gastroenterology and Hepatology, The Brooklyn Hospital Center, Academic Affiliate of The Icahn School of Medicine at Mount Sinai, Clinical Affiliate of The Mount Sinai Hospital, 121 Dekalb Avenue, Brooklyn, New York, USA

Conflict of Interest: None

Correspondence to: Daryl Ramai, MD, Department of Gastroenterology and Hepatology, The Brooklyn Hospital Center, Academic Affiliate of The Icahn School of Medicine at Mount Sinai, Clinical Affiliate of The Mount Sinai Hospital, 121 Dekalb Avenue, Brooklyn, New York 11201, USA

Received 30 October 2017; accepted 13 November 2017; published online 8 January 2018

DOI: https://doi.org/10.20524/aog.2018.0225
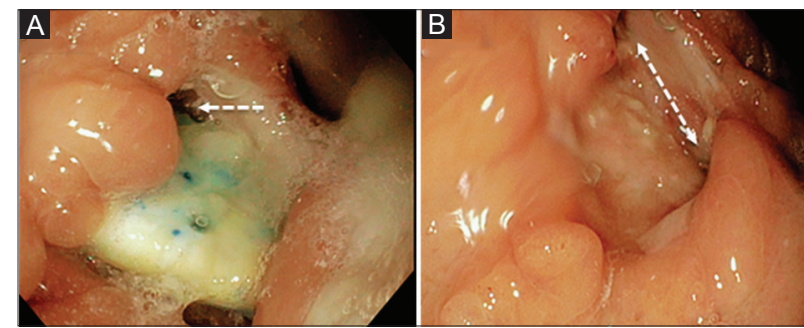

Figure 1 (A) Upper endoscopy revealed stomach perforation by Jackson-Pratt (JP) drain. (B) The JP drain was subsequently withdrawn until it was no longer visible in the stomach lumen
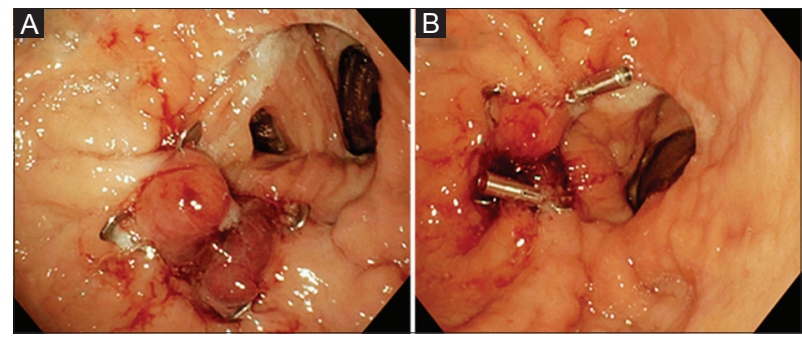

Figure 2 (A, B) The perforation was closed using over the over-thescope clip system

Gastric perforation caused by a JP drain is a rare complication. Risk factors include stiff or non-flexible drains, a faulty staple/suture line, long-term placement, and edema at the surgical site. This case is noteworthy in illustrating how the OTSC technique can be reliably used for the endoscopic closure of perforations. Additionally, OTSCs can be used to correct fistulas, hemorrhages and other gastrointestinal lesions [1].

\section{Reference}

1. Kirschniak A, Subotova N, Zieker D, Königsrainer A, Kratt T. The over-the-scope clip (OTSC) for the treatment of gastrointestinal bleeding, perforations, and fistulas. Surg Endosc 2011;25:2901-2905. 